\title{
Cardiovascular Nuclear Imaging: Balancing Proven Clinical Value and Potential Radiation Risk
}

\author{
The Cardiovascular Council Board of Directors
}

$\mathbf{T}$ he debate on the potential risk of radiation exposure from diagnostic imaging tests highlights the importance of balancing the demonstrated clinical benefit and the theoretic risk of cardiovascular imaging studies. The Cardiovascular Council of the Society of Nuclear Medicine upholds the responsible application of imaging studies that use radiotracers associated with relatively small amounts of ionizing radiation. Radionuclide-based cardiac imaging studies, including myocardial perfusion imaging (MPI), provide accurate diagnostic and prognostic information about patients with suspected or known heart disease. There is a large body of scientific evidence on the clinical value of MPI, based on studies performed on many thousands of patients. On the basis of this information, appropriate-use criteria and guidelines were developed and endorsed by the Society of Nuclear Medicine and other professional societies, including the American College of Cardiology, American Heart Association, and American Society of Nuclear Cardiology (1).

Cardiovascular nuclear medicine studies provide highly sensitive and specific tests that may be indicated for the evaluation of diagnosis, prognosis, and treatment response of coronary artery disease, as well as for selection of patients who benefit from revascularization. The value and justification of MPI for risk assessment is based on large observational outcome studies that demonstrate accurate risk stratification with radionuclide-based MPI in populations with an intermediate pretest risk. The incremental prognostic value of SPECT MPI is greater than that of the exercise electrocardiography stress test or coronary angiography. The cost effectiveness of MPI as a gatekeeper to coronary angiography has been established after being carefully and extensively studied.

Several recent publications have raised concern about the potential harmful effects of ionizing radiation associated with cardiac imaging. Review of the measurement of radiation and associated biologic effects can help put this issue into reasonable perspective. Radiation effective dose is a measure used to estimate the biologic effects of radiation. Measuring the radiation effective dose associated with di-

Received Mar. 17, 2011; revision accepted Mar. 24, 2011.

For correspondence or reprints contact: Mehran M. Sadeghi, Yale University School of Medicine, 950 Campbell Ave., West Haven, CT 06516.

E-mail: mehran.sadeghi@yale.edu

COPYRIGHT @ 2011 by the Society of Nuclear Medicine, Inc.

DOI: 10.2967/jnumed.111.090654 agnostic imaging is complex and imprecise and often results in varying estimates among experts (2). A typical effective dose for a rest-stress same-day SPECT scan using ${ }^{99 \mathrm{~m} T c-}$ labeled agents $(1,110 \mathrm{MBq}[30 \mathrm{mCi}]$ stress, $370 \mathrm{MBq}[10$ $\mathrm{mCi}$ ] rest), the most commonly used MPI protocol, is approximately $10 \mathrm{mSv}$. Other agents and protocols are associated with a wide range of radiation exposure (2). In comparison, exposure to radiation from natural sources amounts to approximately $3 \mathrm{mSv}$ annually. The risk of a fatal malignancy from medical imaging-related radiation is difficult to estimate precisely but is likely small and difficult to discern from the background risk of natural malignancies. The theoretic lifetime attributable risk of cancer from a rest and stress ${ }^{99 \mathrm{~m}} \mathrm{Tc}$-based MPI study for individuals age $35 \mathrm{y}$ or older is less than 1.5 in 1,000 (3). This risk is less in older patients, who constitute most patients evaluated for coronary artery disease. The estimated risk of fatal malignancy from a typical MPI study is 0.5 per 1,000 individuals, compared with a risk of death from natural cancer of 212 per 1,000 (4).

The potential risk of cancer must be balanced against the risk of death, myocardial infarction, or other morbid vascular events in an appropriately referred population. This risk ranges from $1 \%$ to $10 \%$ or more per year and is orders of magnitude greater than the potential lifetime risk of cancer and death from cancer attributable to cardiovascular nuclear medicine studies. Assessment of risk-to-benefit ratio mandates a good understanding of the clinical characteristics of the patient, including risk factors for coronary artery disease, prior history of coronary artery disease, and left ventricular function. For example, given the substantially higher risk of morbid coronary events or heart failure in patients with left ventricular dysfunction, higher radiation exposure associated with ${ }^{201} \mathrm{Tl}$ or ${ }^{18} \mathrm{~F}$-FDG for radionuclide assessment of viability is readily justifiable. In this context, one must not fail to take into account the risks of missing important diagnostic information by not performing a test (which could potentially influence near-term management and outcomes) because of a theoretic concern about a long-term small risk of malignancy. Similarly, assessment of the significance of radiation exposure risk in population-based studies would be challenging without information on the overall pool from which the patients are selected and how representative they are of the total patient population.

While the potential long-term radiation risk associated with cardiovascular nuclear medicine studies is debated (5), 
the Cardiovascular Council supports adherence to the principle of ALARA (as low as reasonably achievable) in the context of performing the appropriate study to address the clinical question effectively. Before performing an MPI study, we must ensure the appropriateness of the study and use a protocol that delivers the least radiation while maintaining diagnostic accuracy and clinical effectiveness. A review of medical records for old studies and a discussion with the referring clinician about the current study may be prudent. The likelihood that the study being considered will affect the clinical management of the patient should be addressed before testing is performed. Routine periodic follow-up scans of asymptomatic individuals should be avoided. We wish to highlight new opportunities for reducing radiation from MPI through development of innovative hardware and software techniques $(6)$, new imaging protocols (e.g., stress-only imaging (7)), and more widespread use of PET (8). These developments may bring the exposure down to less than $5 \mathrm{mSv}$ for complete rest-stress perfusion studies. In parallel, there is a need for novel tracers with improved diagnostic accuracy and reduced radiation exposure and for clinical translation of potentially transformative novel developments in cardiovascular molecular imaging. The usefulness of alternative diagnostic strategies in comparison with SPECT and PET MPI in selecting and monitoring the effectiveness of treatment strategies will need to be addressed.

In summary, radionuclide MPI can provide scientifically validated, accurate, and in certain cases unique information for management of patients with known or suspected coronary artery disease at risk for major cardiovascular events. The radiation exposure risk associated with radionuclide MPI, albeit small and long term as opposed to the higher and more immediate risk for major cardiovascular events, mandates careful adherence to appropriateness criteria and guidelines developed or endorsed by the Society of Nuclear Medicine, American Society of Nuclear Cardiology, American College of Cardiology, and American Heart Association. With recent developments in technology, there are many opportunities to further reduce radiation exposure and further enhance the benefit-to-risk ratio of this well-established, safe imaging modality.

\section{RECOMMENDATIONS FOR MINIMIZING RADIATION EXPOSURE AND OPTIMIZING THE CLINICAL USE OF RADIONUCLIDE CARDIAC IMAGING}

- Adherence to American College of Cardiology/American Heart Association/American Society of Nuclear Cardiology/Society of Nuclear Medicine appropriate-use criteria for radionuclide imaging is recommended.

- Radionuclide MPI in asymptomatic low-risk or intermediate-risk individuals with an interpretable electrocardiogram should be avoided as a first test.

- Routine use of PET and ${ }^{99 \mathrm{~m} T c-b a s e d ~ S P E C T ~ M P I ~}$ studies instead of protocols with higher radiation exposure should be considered.
- Use of nonradioactive, less expensive modalities (e.g., exercise treadmill test) to identify optimal MPI candidates should be considered.

- Incorporation of stress-only protocols is encouraged.

- Implementation of novel software and hardware with the goal of reducing radiation exposure in accordance with the principle of ALARA is encouraged.

\section{APPENDIX}

The Cardiovascular Council Board of Directors Mehran M. Sadeghi

President

Yale University School of Medicine

New Haven, Connecticut

Ronald G. Schwartz

Vice President

University of Rochester Medical Center

Rochester, New York

Robert S. Beanlands

Vice President-Elect

University of Ottawa Heart Institute

Ottawa, Canada

Mouaz H. Al-Mallah

Intern

Henry Ford Hospital

Detroit, Michigan

Frank M. Bengel

Immediate Past-President

Medizinische Hochschule Hannover

Hannover, Germany

Salvador Borges-Neto

Board Member

Duke University Medical Center

Durham, North Carolina

Elias H. Botvinick

Board Member

University of California-San Francisco

San Francisco, California

Dominique Delbeke

Board Member

Vanderbilt University Medical Center

Nashville, Tennessee

Nancy McDonald Deloatch

Board Member

St. Vincent's Medical Center

Jacksonville, Florida

Marcelo F. Di Carli

Board Member

Brigham and Women's Hospital

Boston, Massachusetts 


\section{Vasken Dilsizian \\ Board Member}

University of Maryland Medical Center

Baltimore, Maryland

Sharmila Dorbala

Board Member

Brigham and Women's Hospital

Boston, Massachusetts

\section{Rory Hachamovitch}

Past President

Cleveland Clinic

Cleveland, Ohio

\section{Zuo-Xiang He}

Board Member

Fu Wai Hospital, CAMS and PUMC

Beijing, China

\section{Diwakar Jain}

Past President

Drexel University College of Medicine

Philadelphia, Pennsylvania

Edward L. Lyons, Jr.

Treasurer

GE Healthcare Medical Diagnostics

Princeton, New Jersey

Robert A. Pagnanelli

Secretary

Duke University Medical Center

Durham, North Carolina

\section{Terrence D. Ruddy}

Board Member

University of Ottawa Heart Institute

Ottawa, Canada

Thomas H. Schindler

Board Member

Hopitaux Universitaires de Geneve

Geneva, Switzerland

\author{
Albert J. Sinusas \\ Past President
}

Yale University School of Medicine

New Haven, Connecticut

\section{Prem Soman}

Board Member

University of Pittsburgh Medical Center

Pittsburgh, Pennsylvania

\section{H. William Strauss}

Board Member

Memorial Sloan-Kettering Cancer Center

New York, New York

Mark I. Travin

Past President

Montefiore Medical Center

Bronx, New York

\section{REFERENCES}

1. Hendel RC, Berman DS, Di Carli MF, et al. ACCF/ASNC/ACR/AHA/ASE/ SCCT/SCMR/SNM 2009 appropriate use criteria for cardiac radionuclide imaging: a report of the American College of Cardiology Foundation Appropriate Use Criteria Task Force, the American Society of Nuclear Cardiology, the American College of Radiology, the American Heart Association, the American Society of Echocardiography, the Society of Cardiovascular Computed Tomography, the Society for Cardiovascular Magnetic Resonance, and the Society of Nuclear Medicine. J Am Coll Cardiol. 2009;53:2201-2229.

2. Einstein AJ, Moser KW, Thompson RC, Cerqueira MD, Henzlova MJ. Radiation dose to patients from cardiac diagnostic imaging. Circulation. 2007;116:12901305.

3. Berrington de Gonzalez A, Kim KP, Smith-Bindman R, McAreavey D. Myocardial perfusion scans: projected population cancer risks from current levels of use in the United States. Circulation. 2010;122:2403-2410.

4. Gerber TC, Carr JJ, Arai AE, et al. Ionizing radiation in cardiac imaging: a science advisory from the American Heart Association Committee on Cardiac Imaging of the Council on Clinical Cardiology and Committee on Cardiovascular Imaging and Intervention of the Council on Cardiovascular Radiology and Intervention. Circulation. 2009;119:1056-1065.

5. Laskey WK, Feinendegen LE, Neumann RD, Dilsizian V. Low-level ionizing radiation from noninvasive cardiac imaging: can we extrapolate estimated risks from epidemiologic data to the clinical setting? JACC Cardiovasc Imaging. 2010;3:517-524.

6. Duvall WL, Croft LB, Godiwala T, Ginsberg E, George T, Henzlova MJ. Reduced isotope dose with rapid SPECT MPI imaging: initial experience with a CZT SPECT camera. J Nucl Cardiol. 2010;17:1009-1014.

7. Chang SM, Nabi F, Xu J, Raza U, Mahmarian JJ. Normal stress-only versus standard stress/rest myocardial perfusion imaging: similar patient mortality with reduced radiation exposure. J Am Coll Cardiol. 2010;55:221-230.

8. Senthamizhchelvan S, Bravo PE, Esaias C, et al. Human biodistribution and radiation dosimetry of ${ }^{82} \mathrm{Rb}$. J Nucl Med. 2010;51:1592-1599. 